\title{
Spectrum of Extra pulmonary Tuberculosis in Oral and Maxillo- Facial Clinic: Two Distinct Varieties
}

\author{
Dr. Aniket A Kansara, Dr.S.M.Sharma, Dr.B Rajendra Prasad, \\ Dr.Ankur Thakral
}

\begin{abstract}
Extrapulmonary tuberculosis is on the increase world over. Tuberculous Lymphadenitis is the commonest form of extrapulmonary tuberculosis. The focus of Tuberculosis(TB) control programme has been on the pulmonary variety, because that is the cause of lot of misory and ill health. Tuberculos cervical lymphadenitis, or scrofula is one of the most common extra-pulmonary manifestations of tuberculosis. Diagnosis of enlarged lymphnode is challenging. A calcified lymph node is indicative of a prior chronic infection involving the node. This paper is to highlight two distinct variety of extrapulmonary tuberculosis.
\end{abstract}

Keywords: extrapulmonary tuberculosis, lymphadenitis, calcification

\section{Introduction:}

Tuberculosis is one of the biggest health challenges the world is facing . Extrapulmonary tuberculosis is on the increase world over. Tuberculous Lymphadenitis is the commonest form of extrapulmonary tuberculosis. The focus of Tuberculosis(TB) control programme has been on the pulmonary variety, because that is the cause of lot of misory and ill health. The extra pulmonary variety is now beginning to emerge from the shadows though TB remains a worldwide threat to humans, which mostly caused by M.Tuberculosis , an infectious and communicable organism.

Tuberculos cervical lymphadenitis, or scrofula, is one of the most common extra-pulmonary manifestations of tuberculosis. Diagnosis of extra-pulmonary tuberculosis has always been a problem which is a protein disease affecting virtually all organs. Clinically it shows a widespectrum of presentation and depends on anatomical site involved and presents a diagnostic challenge.

\section{Extrapulmonary Tuberculosis:}

Tuberculosis infection can spread from lungs to other parts of the body. TB infection that occur outside the lungs are known as Extrapulmoary Tuberculosis, which is most common in people with weakened immune system. Extrapulmonary tuberculosis occurs in more than $50 \%$ of immunocompromised patients, eventhough risk for extrapulmonary tuberculosis with immunosuppresion. ${ }^{[1]}$ In the fully immunocompetent host bacteria are probably destroyed. If some immune deficit is present some may concentrate at a particular site where they may lie dormant for months or years before causing disease. At times bacteria may be coughed from lungs and swallowed, then they may enter the lymphnodes of neck.

\section{Diagnosis :}

Diagnosis of enlarged lymphnode is challenging. Lymphadenopathy may be caused by drug reactions , infections, immunologic disorders, malignancies, and several disorders of uncertain etiology. Extrapulmonary tuberculosis is the result of dissemination of tubercle bacilli from an initial focus in the lungs soon after primary infection. ${ }^{[2]}$ The dissemination is primarily by way of the lympho-hematogenous route , ${ }^{[3]}$ with seeding of virulent tubercle bacilli in almost all of the organs and tissues of the body. Although in most patients, both pulmonary and extrapulmonary lesions heal, clinically subtle granulomas contain tubercle bacilli which can remain viable for decades. ${ }^{[4]}$ Subsequently breakdown of these lesions can lead to reactivation of extrapulmonary disease. Reactivation may be clinically deceptive for the usual features of infection are often absent. Indeed, the infection may be far advanced before any observable symptom occurs. There are reports of clinically unsuspected extrapulmonary tuberculosis diagnosed only during necropsy. ${ }^{[5-7]}$ Thus up to the present time extrapulmonary tuberculosis continous to present as a clinical problem as reflected by reports of cases with atypical clinical and pathological features, including negative chest roentgenograms. ${ }^{[5,8,9]}$

\section{Lymphnode:}

The lymphnodes are considered to be the fortresses that aid immune defences. Lymphnode enlargement is part of our body's normal immune response. The cervical lymph nodes produce specialized immune system cells called lymphocytes that detect and combat pathogens in the body. When an infection 
is present the nodes swell las they produce larger than normal quantities of lymphocytes, The neck mass is often surrounded by mystique in arriving at a diagnosis and treatment. The location of cervical lymph nodes can be divided into six levels .(fig1)1 The level of lymph nodes can be predictive as to the source of of the problem

Level I - Includes submandibular and submental nodes

Level II,III,IV- encompass lymphnodes along the internal jugular vein deep to sternocleidomastoid muscle in the upper middle and lower thirds of the neck respectively .

Level $\mathrm{V}$-contains the nodes in in the posterior triangle these are commonly enlarged in viral infections for e.g. mononocleosis.

Level VI - lies between the carotid sheeth in the anterior triangle and contains the prelaryngeal and pretracheal nodes.

Lymphadenopathy is a term synonymously used with swollen/enlarged lymph nodes.Cervical lymphadenopathy is defined as cervical lymph nodal tissue measuring more than $1 \mathrm{~cm}$ in diameter. Lymph node swelling due to inflammatory disease usually resolves within 4 to 6 weeks. Therefore any nodes which persists beyond two weeks requires further evaluation. Other suspension is lymph nodes more than $1.5 \mathrm{~cm}$ in diameter,firm,rubbery lymph nodes ,matted lymph nodes and nodes that are fixed or that have decreased mobility. Any node with these features requires further evaluation

A calcified lymph node is indicative of a prior chronic infection involving the node.A history of successfully treated tuberculosis is often associated with this calcification and these are usually asymptomatic.It may prent as single or a chain of submandibular or cervical nodes The calcified nodes are palpable as bony ,hard,round,or leniar masses with variable mobility.On a routine panoramic examination these nodes are observed where they may appear below the inferior border of mandible and near the angle of the mandible.

\section{Case report 1:}

A 30 year old female reported to our department with chief complains of swelling in the left lower third of the face and upper neck region and also mild intermittent pain since 20 days. No H/o fever or any other systemic symptoms like anorexia, weight loss, cough. On extraoral examination swelling was firm, tender, overlying skin is non-pinchable and stretched. There was no signs of paresthesia. On intra-oral examination no abnormality was detected. Orthopentomogram was taken which showed normal study. There was no source of odontogenic infection. Patient's chest x-ray showed no abnormality. Blood profile was done which showed raised $\operatorname{ESR}(90 \mathrm{~mm} / \mathrm{hr})$. Fine needle aspiration was done from the swelling which came as non specific inflammatory nature, possibly of salivary glands. Ultrasonography of swelling was done which came as soft tissue tumour or lymphoma and unlikely to be inflammatory mass. PCR was done which showed Mycobacterium avium intracellulare complex(Atypical mycobacteria). So final diagnosis was scrofula (Extrapulmonary Tuberculosis).

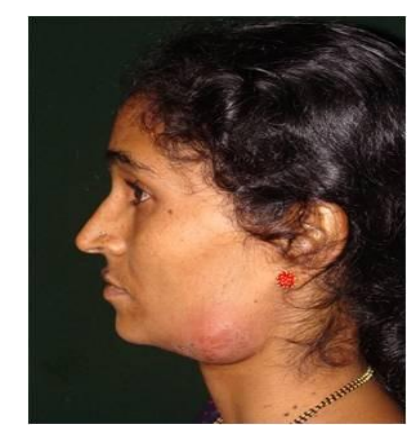

Photo 1.1

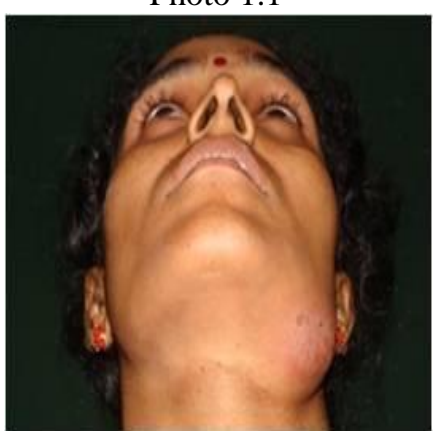

Photo 1.2 

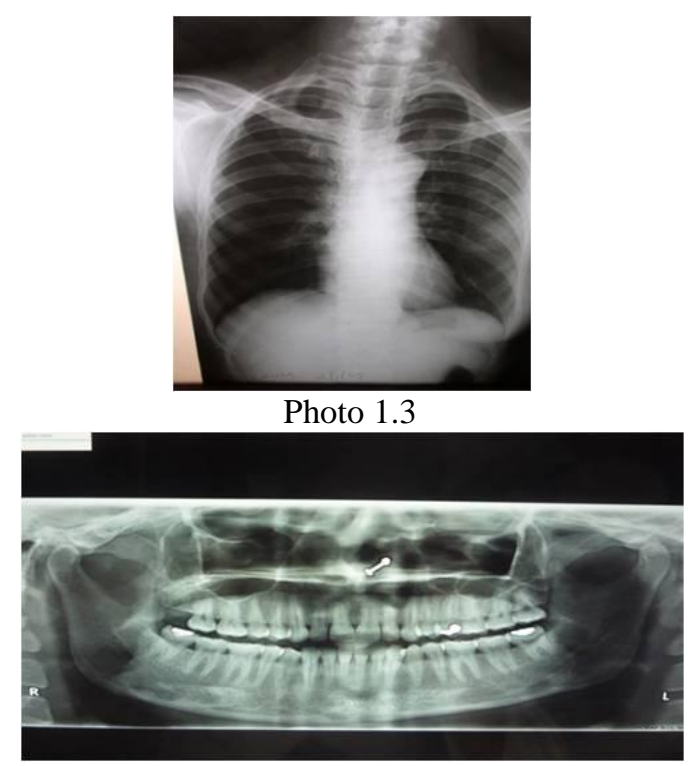

Photo 1.4

\section{Case report 2:}

58 year female maid servent reported with chief complaint of hard swelling over neck patients past medical history revealed she had tuberculosis 20 years ago and treated for lymph node tuberculosis. Patient stated that her treating ESI physician told her that she had lymph node calcification of tuberculos lymphadenopathy and referred her to speciality hospital for further evaluation for which her daughter refused, On orthophantomography $3 \mathrm{~cm} \times 2 \mathrm{~cm}$ Radiopaque mass detected as submandibular lymph node calcification ,almost inferior to mandibular angle. Since patient was aware of condition that was known further examination was not performed and she refused further treatment.

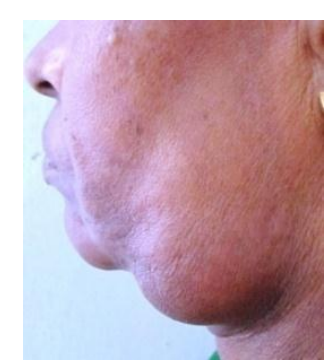

Photo2.1

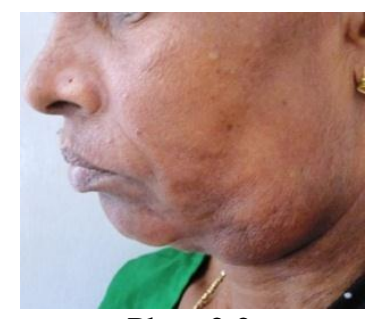

Photo2.2

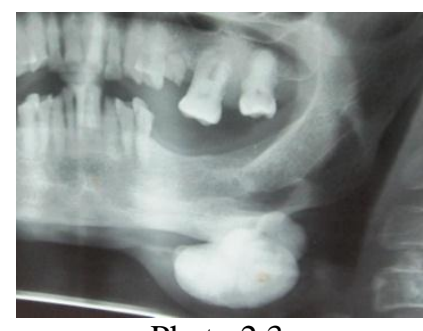

Photo 2.3 


\section{Discussion:}

Tuberculosis is still one of the most significant health care problem worldwide .The disease is gaining importance in some developing countries where it was previously under control.TB is classified as pulmonary and extrapulmonary. Extrapulmonary TB can affect virtually any organ system with some affected more commonly. Lymph node TB was found to be the most prevalent form of extrapumonary TB in many studies ${ }^{[10,11]}$ Milder symptoms in patients whose chest $\mathrm{x}$-rays were negative which may be secondary to the lesser degree of infection of patients with negative chest x-rays.A careful history can provide important clues to the diagnosis of a neck mass

Cervical lymph node calcification is usually asymptomatic and may be found on clinical and radiographic examination Second patient had tuberculosis20 years ago On clinical examination palpable firm to hard node noticed and radiograph revealed calcified nodewhich is regarded as Submandibular cervical lymph node calcification and presumed that TB may be due to ingesting milk infected with Mycobacterium bovies resulting lymph node calcification

Extrapulmonary tuberculosis is usually paucibacillary and any treatment with regimen effective in pulmonary tuberculosisis likely to be effective in the treatment of extrapulmonary tuberculosis as well. Studies are needed to understand the overall proportion of extrapulmonary cases of tuberculosis the anatomical sites and risk factors for extrapulmonary involvement. The need for good quality epidemiological data cannot be overemphasized ${ }^{[12]}$

\section{References}

[1]. Peter Davies, Director Tuberculosis , Research unit, Cardiothoracic centre Liverpool : www.TBalert.org/resourse: An overview Nov $1 / 2005$

[2]. Youmans G.Tuberculosis.Philadelphia : WB Saunders Co.1979

[3]. Simon H , Weinstein A. Genitourinary tuberculosis - clinical features in a general hospital population. Am J Med 1977; 63:410419

[4]. Youmans G. Tuberculosis. In: Hunter G , Swartswerider J (eds). Tropical Medicine , $5^{\text {th }}$ Ed. Pkiladelphia: WB saunders Co, 1976;p.193-211

[5]. Proudfoot AT. Miliary tuberculosis in adults. Br Med J 1969; 2: 273-276

[6]. Jacques J , Sloan JM. The changing pattern of miliary tuberculosis. Thorax 1970;25:237

[7]. Bottiger IE, Nordenstein HN.Disseminated tuberculosis as a cause of fever of obscure origin. Lancet 1962;1:19

[8]. Gricco MN.Acute disseminated tuberculosis as a diagnostic problem - a clinical study based on twenty eight cases. Am Rev Respir Dis 1974; 109: 554-564

[9]. Banner A. Tuberculosis - clinical aspects and diagnosis. Arch Inter Med 1979;139:1387-1390

[10]. Talukder MS,HAQINH, Haque A, Sarker CB, Extrapulmonary tuberculosisin surgical speciality, mymensingh.med.j 2002

[11]. Dwyer DE,McleodC,Collymon PJ,SorrelTC,Extrapulmonary TB a continuing problem in Australia:Australia Nuz.J.Med 1987; $17: 507-511$

[12]. Lalit Kant : Extra-pulmonary tuberculosis: Coming out of the shadows :Indian J Tuberculosis 2004;51:189-190.

Legends:

Photo 1.1 - case report 1 patient's lateral profile

Photo 1.2 - case report 1 patient's front profile

Photo 1.3 - case report 1 patient's chest X-ray

Photo 1.4 - case report 1 patient's OPG

Photo 2.1 - case report 2 patient's lateral profile

Photo 2.2 - case report 2 patient's lateral profile

Photo 2.3 - case report 2 patient's OPG 